INPLASY

PROTOCOL

To cite: Ma et al. The effectiveness of acupoint herbal patching for nonspecific low back pain: A Protocol for systematic review and meta-analysis. Inplasy protocol 202210040. doi: 10.37766/inplasy2022.1.0040

Received: 09 January 2022

Published: 09 January 2022

Corresponding author:

Tianshu Ma

51087482@qq.com

Author Affiliation: Changchun University of Chinese Medicine.

Support: The Jilin province department.

Review Stage at time of this submission: The review has not yet started.

Conflicts of interest: None declared.

\section{The effectiveness of acupoint herbal patching for non-specific low back pain: A Protocol for systematic review and meta-analysis}

\section{Ma, T1; Liu, W2; Dong, Y3; Cheng, L4; Wang, Z5; Liu, X6; Li, T7;} Liu, C. $^{8}$.

Review question / Objective: Non-specific low back pain (NSLBP) is related to disability and work absence and accounts for high economical costs in global.It is a problem that has a negative impact on physical, mental health of patients and quality of life. At present, acupoint herbal patching(AHP) as an adjuvant therapy is currently undergoing clinical trials in different medical centers. This study aimed to design a systematic review and meta-analysis to explore the effects of AHP on non-specific low backpain (NS-LBP).

Condition being studied: We will collect randomized controlled trials (RCTs) to evaluate clinical effectiveness, functional outcomes, quality of life, and side effects of AHP on LBP for systematic review and meta-analysis.RCTs comparing AHP for FC with no treatment, placebo, or conventional drugs will be included.All eligible trials will be included regardless of language and publication type. RCTs that meet the requirements will be included for data mining. Articles of the following research types will be excluded: case series, observational studies (including cohort studies and casecontrol studies) and retrospective studies, qualitative studies, animal experiments, review articles. In addition, there will be no restrictions on study area, race, patient gender.

INPLASY registration number: This protocol was registered with the International Platform of Registered Systematic Review and Meta-Analysis Protocols (INPLASY) on 09 January 2022 and was last updated on 09 January 2022 (registration number INPLASY202210040).

\section{INTRODUCTION}

Review question / Objective: Non-specific low back pain (NS-LBP) is related to disability and work absence and accounts for high economical costs in global.It is a problem that has a negative impact on physical, mental health of patients and quality of life. At present, acupoint herbal patching(AHP) as an adjuvant therapy is 
currently undergoing clinical trials in different medical centers. This study aimed to design a systematic review and metaanalysis to explore the effects of AHP on non-specific low backpain (NS-LBP).

Condition being studied: We will collect randomized controlled trials (RCTs) to evaluate clinical effectiveness, functional outcomes, quality of life, and side effects of AHP on LBP for systematic review and meta-analysis.RCTs comparing AHP for FC with no treatment, placebo, or conventional drugs will be included.All eligible trials will be included regardless of language and publication type. RCTs that meet the requirements will be included for data mining. Articles of the following research types will be excluded: case series, observational studies (including cohort studies and case-control studies) and retrospective studies, qualitative studies, animal experiments, review articles. In addition, there will be no restrictions on study area, race, patient gender.

\section{METHODS}

Participant or population: Patients with non-specific low back pain. There will be no restrictions on study area, race, patient gender.

Intervention: Participants in the intervention group are those undergoing acupoint herbal patching, regardless of herbal regimen, acupoints selected, patching time. There will not be any restrictions on age and original countries of the participants.

Comparator: In the control group, patients received medication, no treatment, sham or placebo acupoint catgut embedding, acupuncture/electro-acupuncture and etc. The other interventions between the control group and the intervention group should be the same.

Study designs to be included: For inclusion, at least one of the following outcome measures should have been measured in the RCT: pain intensity [e.g., visual analog scale (VAS), numerical rating scale (NRS), McGill pain questionnaire], back-specific functional status (e.g., Roland-Morris Disability Questionnaire, Oswestry Disability Index), perceived recovery(e.g., overall improvement), and return to work (e.g., return to work status, sick leave days). The primary outcomes for this review were pain and functional status.

Eligibility criteria: Participants in the intervention group are those undergoing acupoint herbal patching, regardless of herbal regimen, acupoints selected, patching time. There will not be any restrictions on age and original countries of the participants. In the control group,patients received medication, no treatment, sham or placebo acupoint catgut embedding, acupuncture/electroacupuncture and etc. The other interventions between the control group and the intervention group should be the same.

Information sources: An electronic search will be conducted. We will identify relevant studies from the Cochrane Central Register of Controlled Trials,PubMed, Embase, the Web of Science, the Chinese Biomedical Literature Database (CBM), the Chinese Scientific Journal Database (CSJD), the Wan-Fang Database (Wanfang) and the China National Knowledge Infrastructure (CNKI) from their inception to 15 January 2021.

Main outcome(s): For inclusion, at least one of the following outcome measures should have been measured in the RCT: pain intensity [e.g., visual analog scale (VAS), numerical rating scale (NRS), McGill pain questionnaire], back-specific functional status (e.g., Roland-Morris Disability Questionnaire, Oswestry Disability Index), perceived recovery(e.g., overall improvement), and return to work (e.g., return to work status, sick leave days). The primary outcomes for this review were pain and functional status.

Quality assessment / Risk of bias analysis: Review authors will independently evaluate each included study and will follow the 
domain-based evaluation as developed by the Cochrane Handbook for Systematic Reviews of Interventions. They will assess the following domains: 1. selection bias (random sequence generation and allocation concealment), 2. performance bias (blinding of participants and personnel), 3. detection bias (blinding of outcome assessment), 4. attrition bias (incomplete outcome data), 5. reporting bias (selective reporting), 6. other bias (such as pre-sample size estimation, early stop of trial). Each domain will be divided into 3 categories: "low risk", "high risk", or "unclear risk".

Strategy of data synthesis: We will analyze the data with RevMan software (Version 5.3) (Available at: https: / / community.cochrane.org/help/tools-andsoftware/revman-5) provided by The Cochrane Collabora-tion.A meta-analysis using random or fixed effects models will be conducted to summarize the data. Continuous data will be pooled and presented as mean differences or standardized mean difference with their 95\% Cl. Dichotomous data will be pooled and expressed as risk ratio with their $95 \%$ Cl. We will interpret it using the following criteria: 2 values of $25 \%$ is considered low levels of heterogeneity, $50 \%$ indicated moderate levels, and $75 \%$ indicated high levels.Since low or moderate heterogeneity suggests little variability among these studies, the data will be analyzed in a fixedeffects model. When significant heterogeneity occurs among the studies (P50\%), a random effect model will be performed to analyze the data.

Subgroup analysis: Subgroup analysis will be conducted to evaluate the specific influence of intervention type, age, course of disease, treatment duration on pooled results. If the data is insufficient, qualitative synthesis will be conducted instead of quantitative synthesis.

Sensitivity analysis: In addition, sensitivity analysis will be performed to examine the robustness of the results by eliminating low quality trials. We willalso use Spass software(Version19.0)
Country(ies) involved: China.

Keywords: acupoint herbal patching, nonspecific low back pain, low back pain, protocol, systematic review.

Contributions of each author:

Author 1 - Tianshu Ma.

Author 2 - Wu Liu.

Author 3 - Yan Dong.

Author 4 - Lei Cheng.

Author 5 - Ziyuan Wang.

Author 6 - Xiaona Liu.

Author 7 - Tie Li.

Author 8 - Chengyu Liu. 